\title{
Composition et message de la péricope de la femme adultère (Jn 7,53-8,11)
}

\section{Jacek Oniszczuk}

Traducteur : Roland Meynet

\section{(2) OpenEdition Journals}

\section{Édition électronique}

URL : https://journals.openedition.org/rhetorique/488

DOI : $10.4000 /$ rhetorique.488

ISSN : 2270-6909

Éditeur

UGA Éditions/Université Grenoble Alpes

Édition imprimée

ISBN : 978-2-84310-343-8

Référence électronique

Jacek Oniszczuk, "Composition et message de la péricope de la femme adultère (Jn 7,53-8,11) », Exercices de rhétorique [En ligne], 8 | 2017, mis en ligne le 25 janvier 2017, consulté le 28 novembre 2021. URL : http://journals.openedition.org/rhetorique/488 ; DOI : https://doi.org/10.4000/rhetorique. 488

Ce document a été généré automatiquement le 28 novembre 2021.

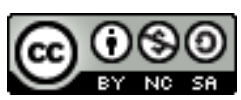

Les contenus de la revue Exercices de rhétorique sont mis à disposition selon les termes de la Licence Creative Commons Attribution - Pas d'Utilisation Commerciale - Partage dans les Mêmes Conditions 4.0 International. 


\section{Composition et message de la péricope de la femme adultère (Jn $7,53-8,11)$}

Jacek Oniszczuk

Traduction : Roland Meynet

\section{NOTE DE L'ÉDITEUR}

Pour agrandir les images, cliquer en-dessous sur les mentions « Agrandir » (taille moyenne) ou «Original»(taille réelle).

1 La péricope qui rapporte l'histoire de la femme adultère est bien connue, surtout à cause de son message qui met en évidence la grande miséricorde de Dieu, laquelle se manifeste dans la personne de Jésus. Pourtant, les exégètes modernes y ont consacré des fleuves d'encre surtout pour d'autres raisons: ce sont d'abord les problèmes historico-critiques de l'authenticité du texte de Jean et de sa place dans le canon, c'est aussi la signification du geste mystérieux de Jésus qui écrit avec son doigt sur le sol. Parmi les problèmes historico-critiques, le plus important est celui que soulève la critique textuelle qui, avec la majorité des chercheurs, affirme qu'à l'origine la péricope n'appartenait pas à l'évangile de Jean ${ }^{1}$. C'est pourquoi certains commentaires importants ne la considèrent même pas ${ }^{2}$, ou s'ils la considèrent, ils ne la font pas remonter à Jésus et jugent qu'elle ne peut être comptée parmi les textes d'«une tradition communautaire de style juif ${ }^{3} »$. Toutefois, la majorité des exégètes fait valoir à juste titre que le récit a toutes les caractéristiques d'une ancienne tradition sur Jésus ${ }^{4}$, reprenant souvent les mots de Wilhelm Heitmüller, qui appelle la péricope une «perle égarée de la tradition antique ${ }^{5} »$. 
2 Le présent article se propose d'aborder le texte de Jn 7,53-8,11, en utilisant pour la première fois l'analyse rhétorique biblique et sémitique, non pas tant pour discuter les questions historico-critiques mais pour relever le défi d'interpréter le texte tel qu'il est maintenant dans le canon des Écritures, grâce à une analyse approfondie de sa composition. Le problème textuel dont il a été question aide, d'une manière très claire et objective, à délimiter le texte analysé ${ }^{6}$, et il sera repris brièvement dans la rubrique Texte, la première étape de la méthodologie utilisée ici. Une plus grande attention sera toutefois accordée à la composition de la péricope qui, avec les considérations sur son contexte biblique, permettront enfin d'aborder certaines questions d'interprétation, en particulier à propos du geste mystérieux de Jésus. Ainsi, le parcours exégétique suivi dans cette étude correspond aux principales étapes de l'analyse rhétorique biblique et sémitique (Texte, Contexte biblique et Interprétation), telle qu'elle est décrite dans le Traité de Roland Meynet ${ }^{7}$.

\section{Texte}

$7,{ }^{53} \mathrm{Et}$ ils s'en allèrent chacun chez soi. $8,{ }^{1}$ Quant à Jésus, il alla au mont des Oliviers. ${ }^{2}$ Mais, dès l'aurore, de nouveau il fut là dans le Temple, et tout le peuple venait à lui, et s'étant assis il les enseignait. ${ }^{3}$ Or les scribes et les Pharisiens amènent une femme surprise en adultère et, la plaçant au milieu, ${ }^{4}$ ils disent à Jésus : " Maître, cette femme a été surprise en flagrant délit d'adultère. ${ }^{5}$ Or, dans la Loi, Moïse nous a prescrit de lapider ces femmes-là. Toi donc, que dis-tu ? " ${ }^{6}$ Ils disaient cela pour le mettre à l'épreuve, afin d'avoir matière à l'accuser. Mais Jésus, se baissant, se mit à écrire avec son doigt sur le sol. ${ }^{7}$ Comme ils persistaient à l'interroger, il se redressa et leur dit: "Que celui d'entre vous qui est sans péché lui jette le premier une pierre ! ${ }^{8}$ Et se baissant de nouveau, il écrivait sur le sol. ${ }^{9}$ Mais eux, entendant cela, s'en allèrent un à un, à commencer par les plus vieux; et il fut laissé seul, avec la femme toujours là au milieu. ${ }^{10}$ Alors, se redressant, Jésus lui dit: « Femme, où sont-ils? Personne ne t'a condamnée ?» ${ }^{11}$ Elle dit : «Personne, Seigneur.» Alors Jésus dit : « Moi non plus, je ne te condamne pas. Va, désormais ne pèche plus. ${ }^{8}$

\section{Questions textuelles}

3 Pour les 183 mots que compte la péricope le texte grec présente plus de 80 variantes différentes 9 . La plupart sont des gloses ou des additions qui cherchent à expliquer certaines rugosités du récit ${ }^{10}$, mais qui, du point de vue de la critique textuelle, ne représentent pas de réel problème, car elles se trouvent dans des manuscrits relativement peu nombreux ou de peu d'autorité. On peut donc raisonnablement omettre de les analyser ; en revanche, il est nécessaire de traiter, même brièvement, de la question de l'authenticité johannique de la péricope ${ }^{11}$.

La preuve textuelle contre l'appartenance de la péricope au texte original du quatrième Évangile est très claire. Pour ce qui est de la preuve externe la péricope est absente des manuscrits indépendants les plus anciens ${ }^{12}$ et chez les Pères de l'Église jusqu'au quatrième siècle. Le témoin le plus ancien qui relate la péricope est le codex de Bèze $\left(V^{\mathrm{e}}\right.$ siècle) ; viennent ensuite de nombreux manuscrits grecs, la majorité de la tradition de la Vieille latine et de la Vulgate. Cependant, le récit est déjà mentionné dans la Didascalia apostolorum (un document de l'Église syrienne du III ${ }^{\mathrm{e}}$ siècle) et sa canonicité est assidûment défendue par les Pères latins (Ambroise, Augustin et Jérôme). L'examen de la preuve interne conduit à une conclusion similaire, soit à cause des 
caractéristiques du vocabulaire non johannique utilisé13 ${ }^{13}$, soit à cause d'une certaine interruption dans la continuité de la narration de 7,1 à 8,59. En outre, la position de la péricope dans les manuscrits n'est pas constante. Bien que la majorité la rapporte en Jn $7,53-8,11$, certains la mettent après Jn 7,36 ou à la fin de Jean, après Luc 21,38 ou à la fin de Luc.

En conclusion, bien que l'authenticité johannique de la péricope soit probablement à exclure, toutefois, étant donné les anciens témoignages ecclésiastiques et les caractéristiques de son texte ${ }^{14}$, ainsi que son message décidément évangélique, il n'est guère possible de douter de sa valeur canonique : il s'agit bien d'un récit qui provient de l'ancienne tradition orale sur Jésus ${ }^{15}$.

\section{Questions grammaticales et lexicographiques}

En 8,6 le geste énigmatique de Jésus est exprimé par l'expression grecque tō $\bar{o}_{i}$ daktylo $\bar{o}_{i}$ kategraphen eis tēn gēn ( «avec le doigt il écrivait sur la terre »). Le verbe katagraphō est un verbe composé (kata + graphō), dont la racine signifie à l'origine " graver " $(1 \mathrm{R} 6,28$; Is 22,16 ; Dt 27,3 ; dans la Septante [LXX] ${ }^{16}$ ) ou plus généralement «enregistrer » ou "dessiner ", mais dans le Nouveau Testament (NT) il se réfère le plus souvent à l'« écriture ${ }^{17}$ ». L'acte d' ' écrire avec le doigt » est très rare dans la Bible et se réduit pratiquement à trois événements : outre celui de Jn 8,6, il n'apparaît que dans le cas des tables de pierre, confiées à Moïse sur le mont Sinaï, «écrites par le doigt de Dieu » (gegrammenas to $\bar{o}_{i}$ daktylo $\bar{o}_{i}$ tou theou; Ex 31,18; voir aussi Dt 9,10 dans la $\mathrm{LXX}^{18}$ ) et dans le récit de l'écriture sur le mur du palais du roi Balthazar, faite par « les doigts d'une main d'homme » (daktyloi cheiros anthrōpou, Dn 5,5). L'acte d'« écrire sur la terre » n'apparaît que dans un texte de Jérémie, où le prophète menace ceux qui se détournent du Seigneur d'" être écrits sur la terre" (epi tēs gès graphētōsan; Jr 17,3, LXX; le texte hébreu dit : " seront écrits sur la terre »).

Dans les mots qui suivent le geste « d'écrire sur la terre » Jésus utilise le substantif grec anamartētos, traduit ici par "sans péché » $(8,7)$, un hapax dans le NT (dans la LXX il

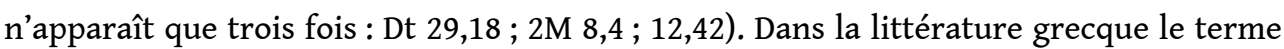
est assez fréquent et signifie généralement "sans défaut ", pas nécessairement dans le sens moral ou religieux, mais le contexte de la scène johannique implique la nonculpabilité devant Dieu, mais sans référence spécifique à la sphère sexuelle ${ }^{19}$. L'utilisation du substantif au singulier et avec l'article défini ne semble pas du tout étrange, car il est impliqué par la construction grammaticale de la phrase ho anamartētos hymōn prōtos ep'autēn baletō lithon), où le " premier » qui jette la pierre ne peut être qu'un seul, mais il n'est pas davantage défini ${ }^{20}$.

8 Au début ce sont « les scribes et les pharisiens » $(8,3)$ qui conduisent la femme adultère à Jésus, mais à la fin ceux qui quittent la scène les premiers sont « les plus vieux » $(8,9)$. L'adjectif comparatif pluriel presbyteroi, qui n'apparaît qu'ici dans Jean, prend souvent dans la tradition juive et chrétienne un sens technique, indiquant une mission (voir Mt $15,2)$ ou un titre désignant les membres d'un organisme officiel, en particulier le Sanhédrin (voir Lc 22,66; 1Tim 4,14 ${ }^{21}$ ). Mais dans Jn 8,9 on doit plutôt exclure le sens technique, parce que le contexte suggère qu'il s'agit ici simplement de gens d'un âge avancé $^{22}$. 


\section{Composition}

9 Le texte ${ }^{23}$ de la péricope, de la taille d'un passage ${ }^{24}$, est composé de trois parties disposées concentriquement précédées d'une introduction $(7,53-8,2)$. Celle-ci fournit les circonstances de l'événement: le temps («à l'aube»), le lieu («le temple») et l'activité de Jésus (« s'étant assis, il enseignait ») :

\begin{tabular}{llll}
\hline $7,{ }^{53}$ Et ILS ALLĖRENT & $\begin{array}{l}\text { chacun } \\
\text { ALLA }\end{array}$ & $\begin{array}{l}\text { dans la maison } \\
\text { au mont }\end{array}$ & $\begin{array}{l}\text { de lui. } \\
\text { des Oliviers. }\end{array}$ \\
$\begin{array}{l}{ }^{2} \text { Mais à l'aube de nouveau } \\
\text { et tout le peuple } \\
\text { et s'étant assis, }\end{array}$ & $\begin{array}{l}\text { il arriva } \\
\text { venait } \\
\text { il enseignait }\end{array}$ & $\begin{array}{l}\text { au temple } \\
\text { à lui } \\
\text { eux. }\end{array}$ \\
\hline
\end{tabular}

10 Le premier segment bimembre oppose l'« aller » $(7,53)$ des protagonistes de la scène précédente (y compris les "pharisiens»; voir 7,47$)$ et l'«aller» de Jésus $(8,1)$, préparant ainsi le conflit, développé plus tard, entre les protagonistes de cette scène et le Maître. Les circonstances de l'événement sont importantes, car dès le début elles montrent la grande autorité de Jésus («s'étant assis il les enseignait» dans le « temple »), qui sera ensuite comparée à celle de « Moïse » et de la « Loi » $(8,5)$.

11 La symétrie du corps du récit autour de la partie centrale $(8,6 \mathrm{c}-8)$ est facilement démontrable grâce à une série de parallélismes formels, mis en évidence dans la réécriture :

- les termes initiaux pour les parties extrêmes : «conduisent » (3a) et «sortaient » (9a) qui indiquent deux mouvements opposés ;

- le substantif «femme », deux fois dans chacune des parties extrêmes (3b.4b; 9d.10b) et l'expression « au milieu » (3c.9d);

- la présence des questions, limitée aux parties extrêmes (5b.10bc);

- la régularité du verbe «dire ", trois fois dans chacune des parties extrêmes (4a.5b.6a; 10a.11a.11c) et une seule dans la partie centrale (7b) ;

- les verbes appartenant au vocabulaire judiciaire « accuser » $(6 \mathrm{~b})$ et « condamner » (10c.11d) ;

- l'opposition sémantique entre « prendre » (3b.4b) et « laisser » (9c).

On notera que la composition des parties extrêmes est similaire : chacune est formée de trois morceaux, et en outre les morceaux centraux se correspondent, en s'opposant. En effet, alors que le premier contient le seul discours des accusateurs qui, se tournant vers Jésus avec le vocatif "Maitre", lui dénoncent le péché de la femme et qu'ils veulent la lapider (4b-5), l'autre rapporte le seul discours de l'«accusée » qui, se tournant vers Jésus avec le vocatif « Seigneur », confirme que personne ne veut plus la condamner (11).

La première partie se focalise sur la question posée à Jésus (5b) ; en effet, les morceaux extrêmes sont narratifs $(3-4 a ; 6 a b)$, tandis que le morceau central, contenant la question, est discursif $\left(4 b-5^{25}\right)$. Dans les deux membres de 5 les accusateurs opposent ce que « Moïse avait ordonné » (5a) à ce que Jésus pourrait « dire » (5b). 


\begin{tabular}{|c|c|c|c|}
\hline $\begin{array}{l}7,53 \text { Et ILS ALLĖRENT } \\
8,{ }^{1,} \text { Mais JÉSUS }\end{array}$ & $\begin{array}{l}\text { chacun } \\
\text { ALLA }\end{array}$ & $\begin{array}{l}\text { dans la maison } \\
\text { au mont }\end{array}$ & $\begin{array}{l}\text { de lui. } \\
\text { des Oliviers. }\end{array}$ \\
\hline $\begin{array}{l}{ }^{2} \text { Mais à l'aube } \\
\text { et tout le peuple } \\
\text { et s'étant assis, }\end{array}$ & $\begin{array}{l}\text { de nouveau } \\
V E N A I T \\
\text { il enseignait }\end{array}$ & $\begin{array}{l}\text { il arriva } \\
\text { à lui } \\
\text { eux. }\end{array}$ & au temple \\
\hline $\begin{array}{l}:{ }^{3} \text { Or CONDUISENT } \\
: \because \text { une FEMEE }\end{array}$ & $\begin{array}{l}\text { les scribes } \\
\text { en adultère }\end{array}$ & $\begin{array}{l}\text { et les pharisiens } \\
\text { PRISE. }\end{array}$ & \\
\hline $\begin{array}{l}: \text { Et placée } \\
:{ }^{4} \text { ils disent }\end{array}$ & $\begin{array}{l}\text { elle } \\
\text { à lui : }\end{array}$ & AU MILIEU, & \\
\hline " MAITRE, cette FEMME & AETE PRISE & sur le fait & faisant-adultère. \\
\hline $\begin{array}{ll}.{ }^{5} \text { Dans la Loi } & \text { Moïse } \\
. . \text { Toi donc } & \text { que }\end{array}$ & $\begin{array}{l}\text { a ordonné } \\
\text { dis-tu? ? }\end{array}$ & de telles & do lapider. \\
\hline $\begin{array}{l}{ }^{6} \text { Or cela } \\
\text { pour }\end{array}$ & $\begin{array}{l}\text { ils disaient } \\
\text { avoir (de quoi) }\end{array}$ & $\begin{array}{l}\text { tentant } \\
\text { ACCUSER }\end{array}$ & $\begin{array}{l}\text { lui, } \\
\text { lui. }\end{array}$ \\
\hline $\begin{array}{l}\text { - Mais JÉSUS } \\
=\text { avec le doigt }\end{array}$ & $\begin{array}{l}\text { en bas } \\
\text { écrivait-en-bas }\end{array}$ & $\begin{array}{l}\text { s'étant penché } \\
\text { sur la terre. }\end{array}$ & \\
\hline $\begin{array}{l}{ }^{7} \text { mais comme } \\
+ \text { il se dressa }\end{array}$ & $\begin{array}{l}\text { ils insistaient } \\
\text { et dit }\end{array}$ & $\begin{array}{l}\text { interrogeant } \\
\text { à eux : }\end{array}$ & lui, \\
\hline $\begin{array}{l}\text { «Le sans-péché } \\
\text { le premier }\end{array}$ & $\begin{array}{l}\text { parmi vous } \\
\text { contre elle }\end{array}$ & jette & une pierre ». \\
\hline $\begin{array}{l}-8 \times \text { Et de nouveau } \\
=\end{array}$ & $\begin{array}{l}\text { s'étant penché-en bas, } \\
\text { il écrivait }\end{array}$ & sur la terre. & \\
\hline $\begin{array}{l}::{ }^{9} \text { Or les entendant (cela) } \\
: \text { en commençant }\end{array}$ & $\begin{array}{l}\text { SORTAIENT } \\
\text { par les plus vieux, }\end{array}$ & un par un, & \\
\hline $\begin{array}{l}:: \text { et FUT LAISSE } \\
: \text { et la FEMME }\end{array}$ & $\begin{array}{l}\text { seul } \\
\text { AU MILIEU }\end{array}$ & étant. & \\
\hline $\begin{array}{l}+10 \text { Mais s'étant dressé } \\
\text { " " FEMME, } \\
\text { Personne }\end{array}$ & $\begin{array}{l}\text { JÉSus } \\
\text { où } \\
\text { toi }\end{array}$ & $\begin{array}{l}\text { dit } \\
\text { sont-ils ? } \\
\text { A CONDAMNEE ?" }\end{array}$ & à elle. \\
\hline $\begin{array}{l}\because:{ }^{11} \text { Mais elle } \\
\text { \& Personne, }\end{array}$ & $\begin{array}{l}\text { dit: } \\
\text { SEIGNEUR ». }\end{array}$ & & \\
\hline $\begin{array}{l}+ \text { Or dit } \\
. \ll \text { Pas même moi }\end{array}$ & $\begin{array}{l}\text { JÉsus: } \\
\text { toi }\end{array}$ & JE CONDAMNE. & \\
\hline $\begin{array}{l}\text { VA, } \\
\text { et désormais }\end{array}$ & ne plus & pèche ". & \\
\hline
\end{tabular}

14 La dernière partie se concentre plutôt sur la réponse de la femme (11), qui réagit à la deuxième question de Jésus $\left(10 \mathrm{c}^{26}\right)$. En effet, les morceaux extrêmes, bien que de taille différente, se correspondent avec la reprise symétrique du verbe « condamner» (10c. 11d) et du nom " Jésus » (10a.11c) qui jouent le rôle de termes médians, et à cause de la régularité des occurrences de « dit » (10a.11a.11c) et des mots similaires, "personne » (oudeis, 10c.11b) et " pas même» (oude, 11d).

15 La partie centrale est importante non seulement parce qu'elle constitue le cœur de la composition, mais surtout parce qu'il se passe quelque chose qui rend possible le renversement inattendu de la situation, grâce auquel la vie de la femme est sauvée. La partie se focalise en effet sur les paroles de Jésus qui se trouvent entre la double mention du geste de "se baisser " (kyptō en 6c et katakyptō en 8a) et «écrire sur la terre » (katagraphō en $6 \mathrm{~d}$ et graphō en $8 \mathrm{~b}$ ).

16 Ainsi, au centre du texte se trouve une double énigme ${ }^{27}$ : l'une liée au sens du geste de Jésus et l'autre à la force de ses paroles ${ }^{28}$ qui réussit à résoudre la tension dramatique de la scène.

17 Si - selon la formule de Paul Beauchamp - la forme est la porte du sens, alors la composition confirme combien il est important d'examiner le geste mystérieux du Maître ${ }^{29}$ afin de saisir le message de toute la scène. En outre, la composition montre que c'est Jésus qui est le protagoniste principal ${ }^{30}$. L'accusation portée contre la femme n'est qu'un prétexte pour lui tendre un piège (6), afin de pouvoir l'accuser et enfin le condamner ${ }^{31}$. Les accusateurs en effet ne parlent pas avec la femme, mais avec Jésus, et n'interrogent pas la femme parce qu'elle, qui est « au milieu » (3c.9d), est simplement l'appât du piège.

18 Les parties extrêmes se correspondent non seulement formellement, mais aussi au niveau du sens et de leur rôle dans le récit. Dans la première partie les personnages actifs sont les accusateurs, la femme ne joue aucun rôle; dans la dernière partie au 
contraire les accusateurs disparaissent de la scène et la femme devient plus active, répondant à Jésus. Il n'est pour eux qu'un "maître » (4b), pour elle au contraire il devient, à la fin de la scène, le "Seigneur » (11b). Ce changement dans la manière de lui adresser la parole peut sembler purement fortuit; néanmoins, il exprime symboliquement le sens profond de la scène, dans lequel sont révélées la véritable identité de Jésus et son autorité, qui dépasse de loin l'autorité de "Moïse ${ }^{32}$ » avec laquelle les accusateurs veulent le comparer (5).

\section{Contexte biblique}

La péricope évoque une série de textes bibliques qui peuvent l'éclairer. Certains sont liés à la question de droit soulevée par les scribes et les pharisiens $(8,4-5)$ sur la façon de punir l'adultère. Pour un tel délit Lv 20,1033 impose la peine de mort, mais sans préciser la façon de l'exécution. De même Dt 22,22 qui traite de manière plus précise de l'adultère, ne le spécifie pas, mais peu après, décrivant le cas de l'infidélité d'une fiancée $^{34}$, est explicitement prévue la mort par lapidation.

Un autre texte concerne les témoignages nécessaires pour les transgressions graves passibles selon la Loi de la peine de mort : Dt 17,1-7. Les deux coupables, l'homme et la femme, doivent être lapidés hors de la ville, sur le témoignage d'au moins deux témoins, qui seront les « premiers » à jeter la pierre (Dt 17,7). Il est intéressant de noter que dans ce passage Jésus n'ordonne pas que la précédence soit accordée aux témoins de l'adultère (qui ne sont même pas mentionnés dans le texte!), mais à celui des accusateurs qui serait « sans péché » (Jn 8,735).

Plus intéressants encore que ces exigences légales auxquelles ne font allusion que quelques versets particuliers de la péricope sont les textes de l'Ancien Testament qui suivent une logique narrative similaire ou ceux qui peuvent jeter quelque lumière sur l'énigme de la partie centrale (Jn 8,6c-836).

\section{L'histoire de Suzanne}

La plupart des chercheurs ont remarqué une certaine similitude entre l'épisode de la femme adultère rapportée dans Jean et l'histoire de Susanne au chapitre 13 du Livre de Daniel. Parmi les points de contact doit être mentionné d'abord le fait que les deux femmes sont accusées du même péché d'adultère, même si dans le cas de Suzanne il est clair dès le début que l'accusation repose sur des mensonges. La peine que risquent les deux femmes est toujours la mort, bien que l'histoire de Daniel ne précise pas le mode d'exécution (Dn 13,28.41.61-62). Les deux femmes sont accusées par les chefs spirituels du peuple (Dn 13,34.45 et Jn $8,3^{37}$ ), qui sont présentés d'une manière très négative (voir Jn 8,6); enfin toutes deux sont sauvées, grâce à la sagesse d'un homme de Dieu (Daniel et Jésus ${ }^{38}$ ).

La péricope de la femme adultère apparaît très semblable à l'histoire de Suzanne, mais cette analogie ne semble pas devoir éclairer le sens du texte de Jean. En particulier, elle ne jette aucune lumière ni sur le geste énigmatique de Jésus qui écrit sur le sol, ni sur la raison de l'extraordinaire puissance de ses paroles. 


\section{Ceux qui « sont écrits sur la terre » $(J r 17,13)$}

Le texte de Jr 17,13 fait partie des oracles contre Juda, qui sont souvent mêlés avec les lamentations du prophète, rejeté par son propre peuple, contre lequel il est appelé à prophétiser. Le verset est souvent cité par ceux qui cherchent à comprendre la signification du geste de Jésus en Jn 8,6.8, car il contient une expression similaire :

Ô espérance d'Israël, Seigneur, que tous ceux qui t'abandonnent restent confus ; que tous ceux qui se détournent de toi soient écrits sur la terre (epi tēs gēs graphētōsan), parce qu'ils ont abandonné le Seigneur, source d'eau vive (Jr 17,13; LXX $\left.{ }^{39}\right)$.

Selon certains chercheurs le geste de Jésus écrivant sur la terre dans la scène de la femme adultère évoquerait cet oracle de Jérémie, comme une sorte d'invitation à la conversion adressée aux impitoyables accusateurs de la femme, pour leur faire comprendre que Dieu seul est juge et que devant lui tous les hommes sont pécheurs ${ }^{40}$. Dans cette perspective justement, le contenu de ce que Jésus écrit n'est pas pertinent pour le récit de Jean - s'il l'était, en effet, il aurait été rapporté par l'auteur - mais il revêt une signification symbolique, comme geste prophétique qui appelle à la conversion.

Pourtant, tout en étant bien situé dans un contexte prophétique, dont Jn 7,53-8,11 n'est pas dépourvu, le verset mentionné semble ne pas avoir d'autre point de contact avec l'histoire de la femme adultère, où la question de la Loi est très prégnante. En outre, il n'éclaire pas la mention du « doigt » avec lequel Jésus écrit sur la terre.

\section{«Les tables de pierre écrites par le doigt de Dieu » (Ex 31,18; voir Dt $9,10)$}

Dans la tradition biblique les tables de l'alliance que Moïse reçut sur le mont Sinaï sont beaucoup plus connues que le seul verset de Jr 17,13. Par conséquent, si, en rapportant le geste de Jésus qui «écrit avec le doigt dans la terre ", l'auteur du récit entendait faire allusion à quelque chose et s'il voulait que l'allusion soit perçue par ses lecteurs, il se serait plutôt référé à ce célèbre événement sur lequel est fondée toute la législation d'Israël ${ }^{41}$. En outre, comme on l'a déjà dit (plus haut, § 6), l'acte d'écrire avec le doigt est très rare dans la Bible et, parmi les textes où il apparaît, les plus proches sont sans aucun doute Ex 31,18 et Jn 8,6. Voici Ex 31,18 :

Quand le Seigneur eut fini de parler avec Moïse sur le mont Sinaï, il lui donna les deux tables du Témoignage, tables de pierre, écrites par le doigt de Dieu (gegrammenas tō ${ }_{i}$ daktylō $\bar{i}_{i}$ tou theou; LXX).

La similitude ne se limite pas à une seule expression, mais couvre un réseau d'éléments qui sont présents dans différentes parties de la péricope de la femme adultère, et aussi dans son contexte actuel du quatrième Évangile. En fait, depuis le début de la péricope sont rapportés des éléments qui sont fondamentaux dans le récit de l'Exode. Dans l'introduction, de même que Moïse était monté sur la montagne du Sinaï et en était redescendu pour transmettre au peuple la Loi qu'il venait de recevoir, ainsi Jésus monte vers la «montagne » des Oliviers $(8,1)$, puis en redescend pour se rendre vers le temple où il est présenté avec autorité («il s'assit et se mit à enseigner »; 8,2). Plus loin les chefs du peuple mettront explicitement l'autorité de Jésus en relation avec celle de « Moïse », dans le contexte de la « Loi » (8,5). Même le verbe «lapider» (lithazein ; 8,5) et le substantif « pierre » (lithos; 8,7 ), qui en grec est de même racine, font référence au 
même matériau que celui des «tables de pierre " (plakes lithinai) décrites dans l'Exode. Bien que dans la péricope de la femme adultère il ne soit pas dit que Jésus a écrit avec le doigt sur la pierre, mais « sur la terre ", il faut noter qu'il est dans la cour du « temple » $(8,2)$, dont le pavement est de pierres! En outre, l'histoire des tables de l'alliance, écrite par le doigt de Dieu, est liée au péché, comme le récit de la femme adultère. En fait, c'est à cause du péché d'idolâtrie (le veau d'or) commis par le peuple qui attendait Moïse au pied du mont Sinaï (Ex 32,1-35) que Dieu a dû écrire une deuxième fois les tables de l'alliance (Ex 34,1). Cependant, en rapportant cette réécriture, la Bible ne dit pas que les nouvelles tables ont été écrites avec le doigt de Dieu; curieusement, dans le récit de la femme adultère non plus, quand le narrateur rapporte la répétition du geste de Jésus pour écrire sur le sol, il ne mentionne plus le doigt (Jn 8,8).

Le parallélisme entre le doigt de Dieu, qui écrivit les tables de la Loi, le «Dieu miséricordieux et compatissant, lent à la colère et riche en bonté et en fidélité » (Ex 34,6 ), et le doigt de Jésus qui sur les pierres du temple écrit la loi de l'amour inconditionnel et du pardon, est en syntonie avec la présentation johannique du Fils, où est montrée non seulement son autorité qui dépasse de loin celle de $\mathrm{Moïse}^{42}$ ou d'Abraham (Jn 8,58) mais aussi sa profonde unité avec le Père (Jn 10,30.38).

\section{Interprétation}

La composition de la péricope justifie le vif intérêt des chercheurs qui tentent de comprendre le sens du geste énigmatique de Jésus. Au centre du texte en effet se trouve la double mention de ce geste, ce qui fait ressortir davantage encore les paroles du Maître qui se trouvent entre deux : «Qui d'entre vous est sans péché jette le premier la pierre contre elle " $(8,7)$. Le geste avec les paroles s'avèrent décisifs pour changer radicalement la situation: ils sauvent la femme de la mort et, au moins pour le moment, libèrent Jésus du piège qui lui avait été tendu.

31 Le centre du texte est donc important, mais sa signification est énigmatique ; elle exige du lecteur un effort d'interprétation notable. Pour ce faire, il est inutile d'essayer de deviner ce que Jésus a écrit; il faut plutôt saisir la puissance allusive de son acte, tel qu'il est décrit par l'auteur du récit. La mémoire biblique qui rappelle les débuts de l'histoire d'Israël, aide à percevoir l'allusion présente dans la scène.

\section{Le doigt de Jésus évoque le doigt de Dieu}

Lorsque «les scribes et les pharisiens » $(8,3)$, connaisseurs de l'Écriture, veulent le comparer avec Moïse duquel ils ont reçu la Loi, le doigt de Jésus écrivant sur le pavement de pierre de la cour du temple renvoie au doigt de Dieu qui écrivit cette même Loi sur des tables de pierre (Ex 31,18). De cette façon, le comportement de Jésus semble indiquer que, même si Moïse avait ordonné de «lapider une femme comme celle-là » $(8,5)$, l'unique auteur de la Loi est Dieu lui-même et, avant de juger la femme, c'est à ce Dieu qu'il faut d'abord se référer, lui qui est « miséricordieux et compatissant, lent à la colère et riche en bonté et en fidélité » $(\mathrm{Ex} 34,6)$. 


\section{Non seulement une pécheresse...} les paroles de Jésus qui se réfèrent au péché $(8,7)$, ainsi entre les deux écritures des tables de la Loi de la part de Dieu (Ex 31,18; Ex 34,1) se trouve l'histoire du péché du peuple : au lieu d'adorer le Dieu qui les a sauvés, ils se prosternent devant le veau d'or qu'ils ont fait de leurs propres mains (Ex 32,1sq.). Ainsi l'attitude de Jésus, qui ne juge personne (Jn 8,15), suggère aux accusateurs de la femme adultère qu'elle n'est pas la seule personne infidèle dans cette assemblée. Eux aussi font partie du même peuple qui adorait le veau d'or, qui, pour utiliser le langage des prophètes, se prostituait avec d'autres dieux, qui fut infidèle à son Dieu de même que la femme qu'ils veulent maintenant condamner avait été infidèle à son mari.

Mais dans sa miséricorde Dieu a pardonné l'infidélité du peuple : il a réécrit les tables de la Loi que Moïse avait brisées (Ex 32,19). C'est peut-être à ce pardon que Jésus fait allusion dans la répétition de son geste. S'il en est ainsi, cette répétition ferait ressortir, davantage encore, l'opposition entre la cruauté des accusateurs de la femme adultère qui ne sont que les gardiens de la Loi, et la miséricorde de Dieu qui est son véritable auteur.

Quoi qu'il en soit, la composition de la péricope confirme cette opposition, et en même temps la ressemblance entre les accusateurs et l'accusée. En effet, la composition met en parallèle leurs discours, lesquels sont placés aux centres des parties extrêmes du passage, tandis que la partie centrale se concentre sur les paroles de Jésus : "Qui d'entre vous est sans péché jette le premier une pierre » $(8,7)$. Ces mots indiquent clairement que les accusateurs sont tous pécheurs, aussi bien que l'accusée. En outre, selon ces paroles, c'est seulement Jésus, le seul «sans péché ", qui pourrait jeter la première pierre, mais il abandonne tout jugement, il ne condamne ni la femme, ni même ses accusateurs, qui l'utilisent parce qu'ils veulent le piéger pour le tuer (Jn 7,25). Contrairement à eux Jésus renonce à une justice qui tue, tout comme Dieu dans l'histoire de l'Exode renonça à faire mourir son peuple infidèle (Ex 32,14).

\section{Le doigt de Jésus est le doigt de Dieu}

Ainsi, la manière dont Jésus est présenté dans la péricope suggère que son attitude est semblable à celle de Dieu. Mais il y a plus : le doigt de Jésus qui écrit sur les pierres du temple, ne fait pas seulement allusion au doigt de Dieu écrivant les tables de la Loi, il révèle aussi une profonde union entre eux (Jn 10,30). Cette façon de présenter Jésus, qui dès le début laisse entrevoir en lui « le Fils de Dieu » (Jn 1,34), est en plein accord avec la façon dont Jésus est présenté tout au long du quatrième évangile ${ }^{43}$. Dans l'Ancien Testament, le but du don de l'Alliance et de la Loi était de donner la vie et non la mort (Dt 30,16). Jésus par son comportement fait référence au plan originel de Dieu, qui est le Dieu des vivants et non des morts (Mc 12,27), au Dieu qui veut que son peuple se convertisse et qu'il vive. Jésus en effet sauve la femme de la mort physique et l'invite à la conversion: "Va, et... ne pèche plus » $(8,11)$. Il n'est pas seulement un "maitre " $(8,4)$, il est vraiment le «Seigneur» $\left(8,11^{44}\right)$ qui a pleine autorité, aussi bien pour interpréter la Loi écrite par son Père, que pour offrir sa miséricorde et son pardon au pécheur, sans condition. 


\section{Non seulement une accusée...}

Encore une fois, la composition du texte permet de remarquer une particularité qui peut offrir un élément de réponse. Les parties extrêmes mettent l'accent non seulement sur l'accusation portée contre la femme pécheresse, mais aussi contre Jésus, le seul homme qui est sans péché. En effet, dans la première partie tous deux se trouvent coincés : la femme est déjà inculpée d'adultère $(8,4-5)$, contre Jésus en revanche on cherche seulement une raison pour l'« accuser » $(8,6)$. Dans la dernière partie tous deux sont libérés : il n'est personne pour condamner la femme $(8,11)$ et pour l'instant Jésus est «laissé seul » et en paix, après le départ silencieux des accusateurs $(8,9)$. Il est intéressant de voir que le texte de l'Évangile de Jean rapproche autant les deux figures de la femme et de Jésus; il indique même que Jésus devra plus tard subir deux tentatives de lapidation (voir Jn 8,$59 ; 10,31$ )!

Quel est le but de ce rapprochement? Si le centre de la péricope fait allusion à l'autorité divine de Jésus, les parties qui l'encadrent semblent faire allusion plutôt à son extrême fragilité humaine, au fait que, dans son Fils, Dieu partage la condition humaine en tout sauf le péché (voir He 4,15). Il la partage jusqu'à la mort physique, dont il a déjà sauvé la femme adultère, pour offrir à tous les pécheurs, elle comprise, la vie éternelle, celle que seul un Dieu fait homme peut donner ${ }^{45}$.

\section{NOTES}

1. Voir B. M. Metzger, A Textual Commentary on the Greek New Testament, Stuttgart, United Bible Societies, 1998, p. 187-189.

2. Voir, par ex., J. Mateos - J. Barreto, El Evangelio de Juan. Análisis lingüístico y comentario exegético, Madrid, Ediciones Cristiandad, 1979, qui n'y consacrent que peu de notes philologiques en appendice de leur commentaire (ibid., p. 925-926). De même B. F. Westcott, J. H. Bernard, E. Hoskyns - N. Davey, C. K. Barrett et T. H. Lightfoot. La péricope est même totalement laissée de côté par exemple par R. Bultmann et H. Strathmann ; voir R. Schnackenburg, Il vangelo di Giovanni, Brescia, Paideia, 1977, II, p. 302.

3. Voir K. Wengst, Il vangelo di Giovanni, Brescia, Queriniana, 2005, p. 331.

4. Voir R. E. Brown, Giovanni. Commento al Vangelo spirituale, Assise, Cittadella, 1999, p. 435 ; voir aussi X. Léon-Dufour, Lecture de l'Évangile selon Jean, II, Paris, Le Seuil, 1990, p. 313-314.

5. Cité par R. Schnackenburg, Il Vangelo di Giovanni, II, Brescia, Paideia, 1977, p. 302.

6. Dans l'édition critique du texte grec de la $28^{\mathrm{e}}$ édition de Nestle-Aland la péricope est mise entre doubles crochets, ce qui indique d'antiques insertions dans la tradition textuelle. L'exacte délimitation du texte est fondamentale pour l'analyse de sa composition.

7. Voir R. Meynet, Traité de rhétorique biblique, Pendé, Gabalda (Rhétorique sémitique 11), 2013. Les limites du présent article ne permettent pas de traiter de façon plus approfondie les questions qui relèvent de la rubrique du Texte (questions de critique textuelle, problèmes grammaticaux et lexicographiques).

8. Traduction de La Bible de Jérusalem, nouvelle édition revue et corrigée (Paris, éd. du Cerf, 1998). 
9. Voir W. Willker, A Textual Commentary on the Greek Gospels. Vol. 4b. The Pericope de Adultera : Jo 7:53 - 8:11, Bremen, 2014, http://www-user.uni-bremen.de/ wie/TCG/index.html [accès : 18.08.2014].

10. Voir par exemple à la fin de 8,8 l'insertion dans peu de manuscrits : henos hekastou autōn tas hamartias ( les péchés de chacun d'entre eux »), qui entend révéler ce que Jésus a écrit par terre. 11. Ce n'est pas non plus le but de cet article de traiter des questions (fort intéressantes mais aussi très complexes) regardant la transmission de la péricope : sa position originelle, par qui, quand et pourquoi elle a été insérée à cet endroit dans l'évangile de Jean, etc.

12. Il faut mentionner surtout les plus anciens papyrus $\left(\mathrm{P}^{66,75}\right)$, les meilleurs codex (Vaticanus et Sinaiticus) et de nombreux manuscrits des anciennes versions (syriaque, sahidique, bohaïrique).

13. Les chercheurs notent que beaucoup de termes n'apparaissent pas dans Jn et sont plus caractéristiques des synoptiques. R. Schnackenburg (Il Vangelo di Giovanni, op. cit., II, p. 304, note 4) observe que «le style non johannique de toute la péricope est si évident qu'on peut renoncer à une démonstration cas par cas [...]».

14. F. Rousseau («La femme adultère. Structure de Jn 7,53-8,11», Biblica 59 [1978], p. 463-480) note que la péricope trahit le style typique de la transmission orale. Metzger aussi (A Textual Commentary, op. cit., p. 188), soutient que le récit possède tous les signes d'une histoire vraie, transmise par la tradition orale de l'Église occidentale et rapportée dans quelques manuscrits.

15. L'omission de la péricope dans les manuscrits les plus anciens peut s'expliquer par la discipline morale des communautés chrétiennes des premiers siècles, préoccupées de lutter contre les péchés sexuels : voir S. A. Panimolle, Lettura pastorale del vangelo di Giovanni, II, Bologne, EDB, 1981, p. 310 ; de même G. M. Burge, «A Specific Problem dans the New Testament Text and Canon: The Woman Caught in Adultery (John 7:53-8:11)», Journal of the Evangelical Theological Society 27 (1984), p. 141-148. Pour G. R. O’Day (« John 7:53-8,11 : A Study in Misreading ", Journal of Biblical Literature 111 [1992], p. 631-640), la raison de la marginalisation de la péricope dans l'Église des premiers siècles furent les préjugés patriarcaux (ibid., p. 640).

16. Voir G. Kittel - G. Bromiley, Theological Dictionary of the New Testament (Abridged), 129, dans BibleWorks 8 [DVD-ROM], Norfolk (VA), 2009-2010.

17. Le sens $d u$ verbe est pertinent pour la discussion sur le fait que Jésus n'était pas analphabète; voir par ex. C. Keith, The Pericope Adulterae, ou P. Foster («Educating Jesus : The Search for a Plausible Context ", Journal for the Study of the Historical Jesus, 4/1 [2006], p. 7-33), qui soutient que Jésus était capable d'écrire ou au moins de lire. Toutefois le verbe n'exclut pas la possibilité qu'ici Jésus dessine quelque chose au lieu d'écrire des lettres; l'ambiguïté se reflète dans certaines traductions (Traduction œcuménique de la Bible: « se mit à tracer du doigt des traits sur le sol »).

18. Noter qu'en Ex 30,18 (Dt 9,10 ajoute la préposition grecque en, « avec »), la formulation est au datif comme en Jn 8,6, bien que le verbe soit légèrement différent. On reviendra plus loin, dans la rubrique du Contexte biblique, sur d'autres ressemblances entre les deux textes.

19. Voir Kittel - Bromiley, Theological Dictionary, op. cit., p. 53.

20. Voir A. Watson («Jesus and the Adulteress », Biblica 80 [1999], p. 101-108), pour qui cet usage implique que Jésus s'adresse ici à une personne précise, c'est-à-dire à l'ex-mari de la femme adultère (ibid., p. 103). L'auteur soutient en effet la thèse que la femme soit remariée et que son second mariage soit adultère, mais seulement selon l'enseignement de Jésus et non selon celui des pharisiens.

21. Voir Kittel - Bromiley, Theological Dictionary, op. cit., p. 932.

22. Voir Schnackenburg, Il Vangelo di Giovanni, op. cit., II, p. 311.

23. La traduction de la réécriture suit à la lettre le texte grec et ne peut donc pas respecter la grammaire du français.

24. Pour une explication détaillée de la terminologie utilisée dans l'analyse rhétorique biblique, voir R. Meynet, Traité, op. cit., chap. 3, « Les niveaux de composition », p. 131-215. 
25. Entre les segments $6 \mathrm{ab}$ et $6 \mathrm{~cd}$ il y a une nette séparation, ce qui justifie leur distribution en deux parties différentes. En effet, $6 \mathrm{ab}$ est plus qu'un simple récit comme en $6 \mathrm{~cd}$ : c'est un commentaire de l'auteur étroitement lié à 4-5 et qui présuppose en outre une profonde connaissance de l'intention des interlocuteurs de Jésus. À noter aussi que cette division permet de respecter le parallélisme étroit entre $6 \mathrm{~cd}$ et $8 \mathrm{ab}$ qui délimite la partie centrale.

26. Si on mettait au centre de la dernière partie tout le verset 10 , au niveau de l'ensemble du passage les parties extrêmes seraient focalisées sur les questions (une en 5 et deux en 10), et en outre on obtiendrait une division plus équilibrée de la dernière partie (un verset pour chaque morceau). Ce faisant, toutefois, on ne respecterait ni la dynamique narrative de 9-11 (deux interventions de Jésus, 10 et 11cde, et une de la femme, 11ab), ni la régularité de la reprise des termes mentionnés ( condamner », « Jésus », « dit », « pas même »/ « personne »). C'est pourquoi nous préférons la composition présentée dans la réécriture, où au centre de 9-11 se trouve la réponse de la femme.

27. C'est un phénomène bien connu de la rhétorique biblique que le centre du texte contienne souvent un élément particulier qui intrigue le lecteur : question, citation, allusion, parabole, ou comme ici une énigme (voir R. Meynet, Traité, op.cit., chap. 8, «Le centre des constructions concentriques », p. 417-469).

28. A. Watson (Jesus and the Adulteress, op. cit., p. 101-102) soutient que la réaction des accusateurs et de la foule est "psychologiquement irréelle », parce que "la réaction normale eut été que chacun saisisse une pierre, non de disparaitre». Réalisme psychologique mis à part, le récit trouve là son point crucial, qui doit être interprété.

29. Ce qui ne signifie pas qu'il faille savoir ce que le Maître a écrit !

30. Son geste et ses paroles occupent le centre du récit, mais ils sont décisifs aussi du point de vue narratif, car ils résolvent le conflit et sauvent la femme de la mort. G. R. O’Day, ( «John 7:538,11: A Study in Misreading ", op. cit., p. 634) a donc raison de dire que la rhétorique du texte n'appuie pas le bipolarisme entre la femme et Jésus, introduit par Augustin avec sa célèbre expression "relicti sunt duo misera et misericordia" (In Johannem 33,5); toutefois dans sa proposition de composition (fondée sur le parallélisme entre 6 b-7 et 8,11 ; ibid., p. 633), où il se concentre sur deux autres pôles, les accusateurs et l'accusée, O’Day ne voit pas le cœur de la scène dans le geste et les paroles de Jésus (6c-8).

31. Peu après, le même évangile décrit une tentative d' "arrêter " Jésus (Jn 8,20), puis deux tentatives de le «lapider» (Jn 8,59 et 10,31), comme dans le cas de la femme. En outre, comme dans la péricope de l'adultère, les tentatives ont lieu près du temple.

32. Voir Ch. P. Baylis («The Woman Caught in Adultery : A Test of Jesus as the Greater Prophet », Bibliotheca Sacra 146 [1989], p. 171-184), qui soutient que la péricope constitue le climax de la présentation de Jésus comme le Prophète dont a parlé Moïse en Dt 18,15, tel qu'elle est exposée dans les sept premiers chapitres de Jean (ibid., p. 172).

33. «L'homme qui commet l'adultère avec la femme de son prochain devra mourir, lui et sa complice ».

34. C'est pourquoi certains pensent que la femme de Jn 8,3-5 est une fiancée infidèle ; cependant déjà J. Blinzler a démontré de manière convaincante qu'il s'agit ici plutôt d'une femme mariée ; voir Schnackenburg, Il Vangelo di Giovanni, op. cit., II, p. 306 ; voir aussi Brown (Giovanni, op. cit., p. 432), qui ajoute le texte d'Ez 16,38-40 comme preuve que « la lapidation était la forme normale de la peine de mort pour tous les types d'adultère ».

35. Le texte est important pour J. D. M. Derrett («Law in the New Testament: The Story of the Woman taken in Adultery ", New Testament Studies 10 [1963-64], p. 1-26), qui soutient l'hypothèse que le mari trahi aurait poussé les témoins à accuser son épouse infidèle. En outre, l'auteur pense que Jésus écrivit sur la terre les mots d'Ex 23,1: «Tu ne prêteras pas la main au méchant en témoignant injustement » (voir Brown, Giovanni, op. cit., p. 433). 
36. Il existe aussi quelques textes du NT qui, par certains côtés, ressemblent à la péricope (par ex., la pécheresse et le pharisien Simon en Lc 7,36-50, passage qui révèle la miséricorde de Jésus, ou bien la question du tribut à César de Mc 12,13-17 et parallèles, qui rapporte comment Jésus se libère habilement d'un piège qui lui était tendu), mais ils semblent moins pertinents, et les limites de cet article ne permettent que de les mentionner.

37. Qu'on se souvienne de la présence du terme presbyteroi (Dn 13,5sqq. et Jn 8,9).

38. Voir Panimolle, Lettura pastorale del vangelo di Giovanni, op. cit., II, p. 307.

39. Le texte hébreu utilise le jussif («qu'ils restent confus » et «qu'ils soient écrits ») au lieu du futur du texte grec («ils resteront confus » et « seront écrits »).

40. Voir Schnackenburg, Il Vangelo di Giovanni, op. cit., II, p. 309. L'auteur appuie cette explication, déjà soutenue par Ambroise, Augustin et Jérôme, parce qu'« elle a le grand avantage de ne pas devoir chercher quelles paroles Jésus a écrit sur la poussière ; mais elle présuppose que ceux qui étaient présents ont pu saisir la signification du geste » (ibid., p. 308). Les autres explications les plus répandues sont : 1) suivant la pratique légale romaine, Jésus aurait écrit pour lui la sentence, avant de la prononcer à haute voix ; 2) Jésus aurait écrit les péchés des accusateurs pour les pousser à ne pas juger la femme; 3) Jésus aurait écrit les paroles d'Ex 23,1b (voir note $35)$; 4) Jésus n'aurait rien écrit, mais son geste aurait servi à exprimer sa réflexion, montrer son attitude imperturbable ou sa volonté de ne pas intervenir (voir ibid., p. 308-309).

41. Il est évidemment plus réaliste de faire cette supposition par rapport à l'intention de l'auteur de la péricope que par rapport à l'intention de Jésus.

42. Dans les dix premiers chapitres de Jean, Moïse est mentionné treize fois, et dans la plupart des cas en référence à Jésus (Jn $1,17.45 ; 3,14 ; 5,45.46 ; 6,32-33 ; 8,5 ; 9,29$ ).

43. Voir, par ex., Jn $1,34.49 ; 3,18 ; 5,25 ; 10,36 ; 11,4.27 ; 19,7 ; 20,31$, pour ne mentionner que les textes où Jésus est identifié comme «le Fils de Dieu ». Cet accord dans la présentation de Jésus conforte l'opinion d'un assez grand nombre d'exégètes que la péricope de la femme adultère, bien qu'insérée après coup en Jn, en fin de compte ne s'harmonise pas si mal ni avec son contexte immédiat ni avec tout le reste de l'évangile.

44. L'usage du vocatif "Seigneur» (grec, kyrie) est ambigu : il peut être une simple marque de politesse (comme notre "Monsieur»), mais il peut aussi faire allusion à une "crainte révérencielle ", à une réaction en face de la sphère divine; voir Schnackenburg (Il Vangelo di Giovanni, op. cit., II, p. 312) qui appelle en outre les paroles de Jésus en 8,11 (« Moi non plus je ne te condamne pas ») « une absolution ».

45. De même X. Léon-Dufour (Lecture de l'Évangile selon Jean, op. cit., II, p. 320), qui suit F. Rousseau (ibid., note 35 ), voit un sens symbolique dans le fait que Jésus «se penche» $(8,6.8)$ et «se redresse » $(8,10)$, comme allusion à son abaissement et à son exaltation salvifiques, par lesquels il réconcilie avec Dieu l'humanité prisonnière du péché.

\section{AUTEURS}

\section{JACEK ONISZCZUK}

Professeur de théologie biblique, chargé du corpus johannique à la Faculté de théologie de l'Université Grégorienne de Rome, membre fondateur et Trésorier de la RBS 